Mauricio M. Victor*, Jorge M. David, Maria C.K. Sakukuma, Elivana L. França

and Anna V.J. Nunes

\title{
A simple and efficient process for the extraction of naringin from grapefruit peel waste
}

https://doi.org/10.1515/gps-2017-0112

Received July 27, 2017; accepted September 22, 2017; previously published online December 6, 2017

Abstract: An improved method to obtain naringin, a bitter flavored flavanone glicoside with proven biological activities, from grapefruit (Citrus $x$ paradisi L.) peel waste is described. The proposed modification of the known process, which involves extraction with methanol and crystallization in water with the addition of dichloromethane, requires shorter processing time and reduced solvent volume. Due to the direct method employed, which did not require the 3-day air-drying stage, the hot extraction of fresh grapefruit albedo using methanol led to higher yields of naringin extract in half the time required. To evaluate the obtained naringin which possessed a wide range of pharmacological properties, it was subjected to chemical transformation into the flavone apigenin, an expensive and naturally-occurring flavonoid obtained in low yields.

Keywords: feedstock for apigenin synthesis; grapefruit waste; naringin extraction.

\section{Introduction}

The citrus juice industry is an important part of the food industry and requires about 25 million tons of produce worldwide in 2016/17. After processing, citrus peels, fruit pulps, and seeds, which comprise almost $50 \%$ of

\footnotetext{
*Corresponding author: Mauricio M. Victor, Departamento de Química Orgânica, Instituto de Química, Universidade Federal da Bahia, Salvador, CEP 40170 115, Brazil; Instituto Nacional de Ciência e Tecnologia/INCT em Energia e Ambiente, Universidade Federal da Bahia, Salvador CEP 40170 115, Brazil; and Centro Interdisciplinar de Energia e Ambiente (CIENAM), Instituto de Química, Universidade Federal da Bahia, Salvador, CEP 40170 115, Brazil, e-mail: mmvictor@ufba.br. http://orcid.org/0000-0003-1236-1973 Jorge M. David, Maria C.K. Sakukuma, Elivana L. França and Anna V.J. Nunes: Departamento de Química Orgânica, Instituto de Química, Universidade Federal da Bahia, Salvador, CEP 40170 115, Brazil; and Instituto Nacional de Ciência e Tecnologia/INCT em Energia e Ambiente, Universidade Federal da Bahia, Salvador, CEP 40170 115, Brazil
}

the original whole fruit mass and generate by-products that are rich in bioactive compounds, are not fully maximized. Focusing on these food industrial wastes, studies have been conducted to isolate bioactive compounds of economic interest from these materials apart from investigations into their typical uses as raw materials for agricultural uses or animal feedings [1].

Apigenin (5,7,4'-trihydroxy-flavone) is a compound distributed in different plants. It is considered as a nutraceutical component with low toxicity and has proven benefits in the health improvement of mammals, despite its limitations in terms of absorption and bioavailability. This flavone exhibits different pharmacological activities, including anti-inflammatory, antioxidant and anticancer activities [2-4]. To date, no natural source can provide this compound with good yields. However, peels from Rutaceae pomelo or grapefruit are known to provide naringin, a flavanone compound, with good yields. Citrus juices can also be considered as an important source of naringin. Specifically, about 77.6-622 $\mu \mathrm{g} \mathrm{ml}^{-1}$ naringenin, the aglicon of naringin, can be found in grapefruit juices [5]. In this context, naringin can be an important synthetic raw material for apigenin synthesis $[6,7]$.

Pomelo is an unusual citrus fruit known as Citrus grandis. Grapefruit (Citrus x paradisi) is the result of crossing the pomelo and the sweet orange (Citrus sinensis). Recently, pomelo peels have been studied as a potential natural source of naringin [8]. This method successfully demonstrated an improvement over the typical procedure used for obtaining flavanone glycoside from citrus pomelo dry peels: $2.4 \%$ yield $(\mathrm{w} / \mathrm{w})$ against direct hot water extraction $(1.12 \% \mathrm{w} / \mathrm{w})$ or other less efficient methods. The addition of dichloromethane during the water crystallization step $(14 \% \mathrm{v} / \mathrm{v})$ can help with the extraction of by-products, which could hinder naringin crystallization. However, for the method to be efficient, the albedo must be air-dried for 3 days, followed by oven-drying overnight, comprising a 4-day substrate preparation period. Moreover, the extraction with methanol requires 3 days and the crystallization step at room temperature requires 3 more days. Thus, the entire process takes 10 days to be completed. Therefore, in this work, we proposed the use of grapefruit peel as a substrate for naringin extraction, along with an alternative method to improve the time and yield aspects 
of the extraction method. We also aimed to demonstrate the transformation of naringin into apigenin - a natural product with a high market value - with good yields.

\section{Materials and methods}

\subsection{Chemicals and instruments}

Commercially available methanol, ethanol, dichloromethane (all solvents from Hexis, Brazil), sulfuric acid (Aldrich Co., USA), and iodide (Merck, Germany) were used without further purification. The pyridine (Aldrich Co., USA) was treated with calcium hydride (Aldrich Co., USA), after which it was distilled and stored over 4A molecular sieves (Aldrich Co., USA). The reactions involving anhydrous solvents were carried out under argon atmosphere. The reactions were monitored by Silica TLC plates (Macherey-Nagel, Germany). ${ }^{1} \mathrm{H}$ NMR spectra were recorded at $400.15 \mathrm{MHz}$ and ${ }^{13} \mathrm{C}$ NMR spectra at $100.04 \mathrm{MHz}$ on a Bruker equipment (Avance). Chemical shifts, given on the $\delta$ (ppm), were referenced to the residual, non-deuterated solvent. Infrared spectra were recorded on a Shimadzu FTIR spectrophotometer model IRAffinity-1S in the ATR mode.

\subsection{Grapefruits}

Fresh grapefruits (Citrus x paradisi L.) were purchased from a local market in Salvador (Bahia), Brazil. These fruits originated from Chile.

\subsection{Grapefruit peels}

The peels were hand-sliced and the albedos (white spongy interior) were separated from the flavedos (orange exterior). For the dry experiments, albedos were cut in small pieces and air-dried for 2 days, after which they were placed in an oven to dry at $40^{\circ} \mathrm{C}$ overnight until constant weight. For the direct experiments, fresh albedos were only cut in small pieces immediately after separation from the flavedos.

\subsection{Naringin extraction}

Naringin isolation from the albedos was carried out by modifying the method described in the literature [8]. The typical method featured methanol extraction followed by crystallization in water. In our tested conditions, methanol was employed to extraction of fresh and dry albedo at room temperature and under heating. Each experiment was repeated three times. Naringin was characterized by the physical and spectrometric data. Such data were compared with the reported data in the literature.

2.4.1 Method A: dry albedo/room temperature methanol extraction: In an Erlenmeyer flask, $190 \mathrm{ml}$ of methanol was added to $30 \mathrm{~g}$ of dry albedo. After 3 days, the slurry was filtered and the methanol was distilled off in a rotary evaporator under reduced pressure at $45^{\circ} \mathrm{C}$. Water $(20 \mathrm{ml})$ was added to the methanolic extract obtained $(3.0 \mathrm{~g})$ and the mixture was stirred at $60^{\circ} \mathrm{C}-70^{\circ} \mathrm{C}$ for $30 \mathrm{~min}$ before being transferred into a separated funnel. Dichloromethane ( $3 \mathrm{ml}$ ) was added and the mixture was transferred to a stoppered flask and left for 4 days at room temperature. The organic layer was removed off and the naringin crystals $(660 \mathrm{mg}, 2.2 \% \mathrm{w} / \mathrm{w})$ were collected by filtration through filter paper and then dried in a vacuum desiccator.

2.4.2 Method B: dry albedo/hot methanol extraction: In an Erlenmeyer flask, $60 \mathrm{ml}$ of methanol was added to $10 \mathrm{~g}$ of dry albedo. The mixture was heated at $55^{\circ} \mathrm{C}$ for $3 \mathrm{~h}$, after which the organic solvent was removed. Next, over $60 \mathrm{ml}$ of methanol was added and new hot extraction was carried out for $30 \mathrm{~min}$. The combined organic phases were dried in a rotary evaporator under reduced pressure at $45^{\circ} \mathrm{C}$. The treatment of methanolic extract with water/dichloromethane and the collection of another crop of naringin crystals $(260 \mathrm{mg}, 2.6 \% \mathrm{w} / \mathrm{w})$ were performed as described above.

2.4.3 Method C: wet albedo/hot methanol extraction: In an Erlenmeyer flask, $330 \mathrm{ml}$ of methanol was added to $50 \mathrm{~g}$ of fresh albedo. The mixture was heated at $55^{\circ} \mathrm{C}$ for $3 \mathrm{~h}$, after which the organic solvent was removed. Then, over $100 \mathrm{ml}$ of methanol was added and the new hot extraction process was carried out. The combined organic phases were dried in a rotary evaporator under reduced pressure at $45^{\circ} \mathrm{C}$. The treatment of methanolic extract with water/dichloromethane and the collection of another crop of naringin crystals (500 mg, 4.1\% w/w based on dry albedo) were performed as described above.

2.4.4 Analytical data for naringin: White solid. M.p. $169.8^{\circ} \mathrm{C}-170.2^{\circ} \mathrm{C}$ [lit. 170.0 $0^{\circ} \mathrm{C}$ ] [9]. IR (ATR, $\mathrm{cm}^{-1}$ ) 3372, 1645, 1632, 1583, 1177, 1038 and 820. ${ }^{1} \mathrm{H}$ NMR (CD $\mathrm{OD}, 400 \mathrm{MHz}$ ): $\delta 7.32$ (d, $J=8.5 \mathrm{~Hz}, 2 \mathrm{H}, \mathrm{H}-2^{\prime}$; H-6'), 6.83 (d, $J=8.5 \mathrm{~Hz}, 2 \mathrm{H}, \mathrm{H}-3^{\prime}$; H-5'), 6.19 (d, J=2.1 Hz, 1H, H-6), 6.16 (d, $J=2.1 \mathrm{~Hz}, 1 \mathrm{H}, \mathrm{H}-8), 5.407-5.36$ (m, 1H, H-2), 5.25-5.24 (m, 1H, H-1'" ), 5.13-5.11 (m, 1H, H-1"), 3.94-3.36 (m, 10H, H-6", H-5"', H-5", H-3"', $\left.\mathrm{H}-3^{\prime \prime}, \mathrm{H}-2^{\prime \prime \prime}, \mathrm{H}-2^{\prime \prime}, \mathrm{H}-4^{\prime \prime \prime}, \mathrm{H}-4^{\prime \prime}\right), 3.17-3.15$ (m, $1 \mathrm{H}, \mathrm{H}-3_{\mathrm{ax}}$ ), 2.76 (dd, J=17.2 and $2.8 \mathrm{~Hz}, 1 \mathrm{H}, \mathrm{H}-3_{\text {eq }}$ ), 1.29 (d, J=6.2 Hz, 3H, H-6"'). Figure 1 shows ${ }^{1} \mathrm{H}$ NMR of naringin obtained from grapefruit albedo.

\subsection{Synthesis of apigenin}

The iodine $(0.90 \mathrm{~g}, 3.6 \mathrm{mmol})$ was added to a solution of extracted naringin $(2.00 \mathrm{~g}, 3.4 \mathrm{mmol})$ in dry pyridine $(20 \mathrm{ml})$. The mixture was heated for $5 \mathrm{~h}$ at $95^{\circ} \mathrm{C}$, cooled to room temperature, and poured into ice. The resulting light-yellow solid was filtered, washed with saturated solution of sodium thiosulfate and water, respectively, and dried in a vacuum to afford $1.71 \mathrm{~g}$ of rhoifolin with an $86 \%$ yield. Alternatively, the product can be extracted using an ethyl acetate/ cold water (3:2) mixture if precipitation does not occur.

The rhoifolin (1.01 g, $1.7 \mathrm{mmol}$ ) was dissolved in ethanol (25 ml) and concentrated $\mathrm{H}_{2} \mathrm{SO}_{4}(3 \mathrm{ml})$ was added dropwise. The mixture was heated under reflux for $3 \mathrm{~h}$, and then cooled to room temperature. Next, $10 \mathrm{ml}$ of ice cold distilled water was added and the $\mathrm{pH}$ was adjusted to 3.0 with the sodium bicarbonate solution. The resulting precipitate was filtered, washed with ice water and dried under vacuum to give $0.41 \mathrm{~g}$ of apigenin as a yellow solid in $86 \%$ yield ( $74 \%$ overall yield).

$\mathrm{Mp}>300^{\circ} \mathrm{C}$ (decomp.). IR ( $\left.\mathrm{cm}^{-1}\right): 3447,1655,1609,1500,1355$, 1246, 1182, 829. ${ }^{1} \mathrm{H}$ NMR (DMSO-d $\mathrm{d}_{6}, 400 \mathrm{MHz}$ ): $\delta 12.95$ (s, $1 \mathrm{H}, \mathrm{OH}-5$ ), 


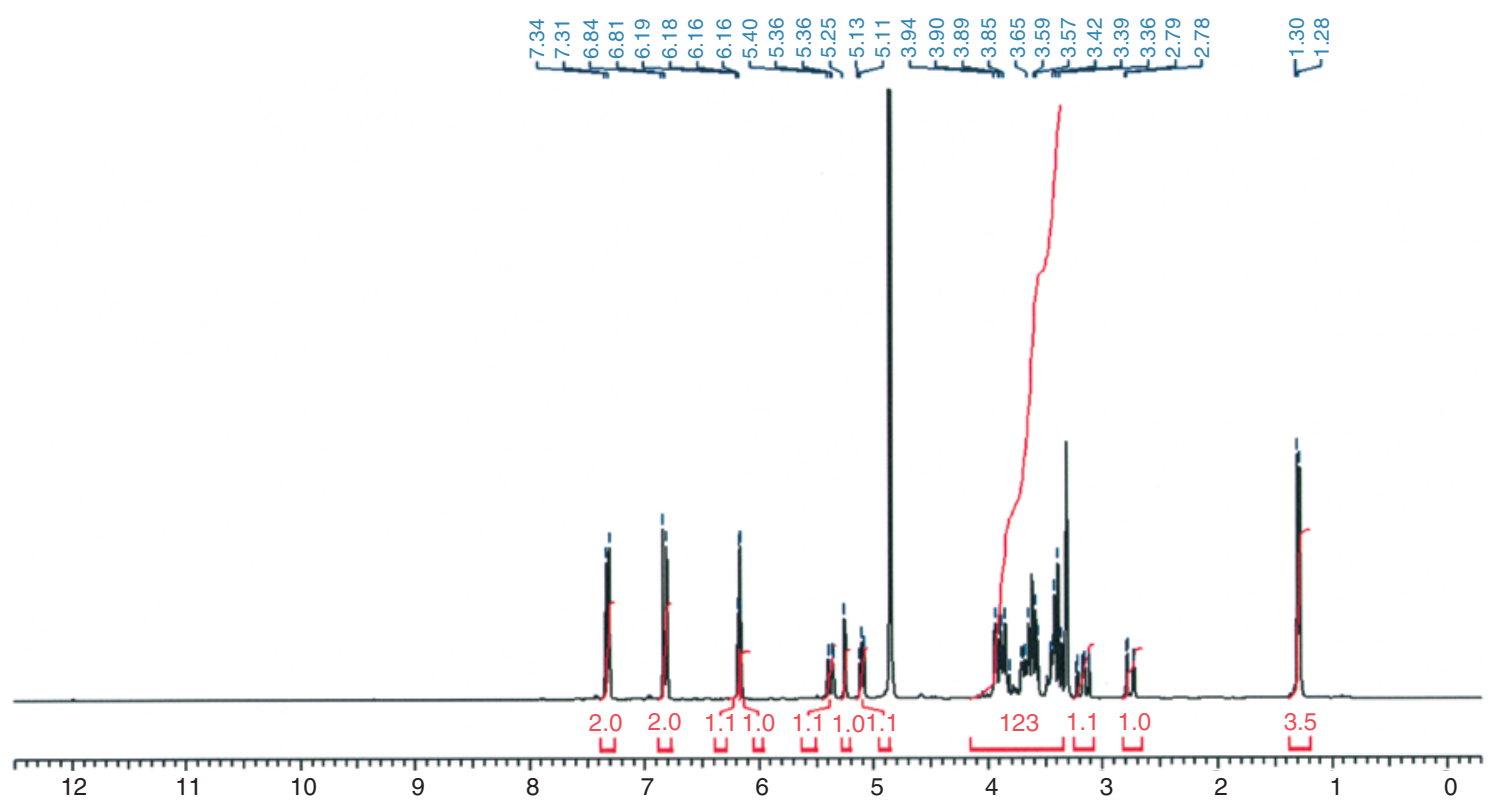

Figure 1: The ${ }^{1} \mathrm{H} N M R\left(\mathrm{CD}_{3} \mathrm{OD}, 400 \mathrm{MHz}\right)$ of extracted naringin.

12.24-8.27 (br, 2 H, OH-4'; OH-7), 7.91 (d, $J=8.7 \mathrm{~Hz}, 2 \mathrm{H}, \mathrm{H}-2^{\prime}$; H-6'), 6.92 (d, $J=8.5 \mathrm{~Hz}, 2 \mathrm{H}, \mathrm{H}-3^{\prime}$; H-5'), 6.75 (s, $1 \mathrm{H}, \mathrm{H}-3$ ), 6.46 (s, $1 \mathrm{H}, \mathrm{H}-8$ ), 6.17 (s, $1 \mathrm{H}, \mathrm{H}-6) .{ }^{13} \mathrm{C}$ NMR (DMSO-d, $100 \mathrm{MHz}$ ): $\delta 181.7$ (C-4), 164.5 (C-7), 163.7 (C-2), 161.4 (C-5), 161.2 (C-9), 157.3 (C-4'), 128.4 (C-2'; C-6'), 121.1 (C-1'), 116.0 (C-3'; C-5'), 103.5 (C-10), 102.8 (C-3), 98.9 (C-6), 94.0 (C-8). The ${ }^{1} \mathrm{H}$ and ${ }^{13} \mathrm{C}$ NMR spectra of synthesized apigenin are described in Figures 2 and 3, respectively.

\section{Results and discussion}

In the first approach, we decided to validate the method described in the literature due to the exchange of species involved in the extraction of naringin, moving from pomelo to grapefruit. Although these species are

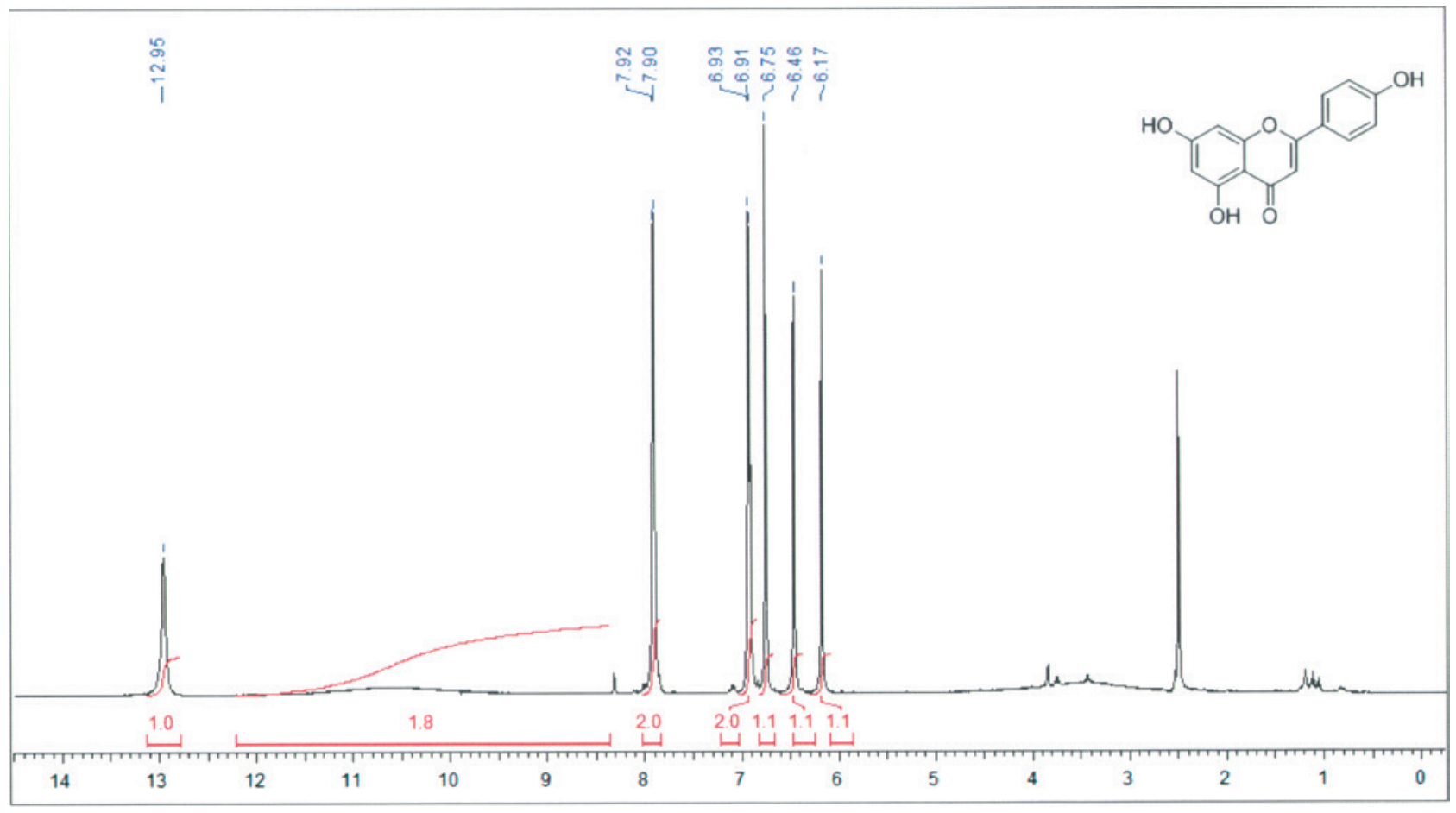

Figure 2: The ${ }^{1} \mathrm{H}$ NMR (DMSO- $\mathrm{d}_{6}, 400 \mathrm{MHz}$ ) of synthesized apigenin. 


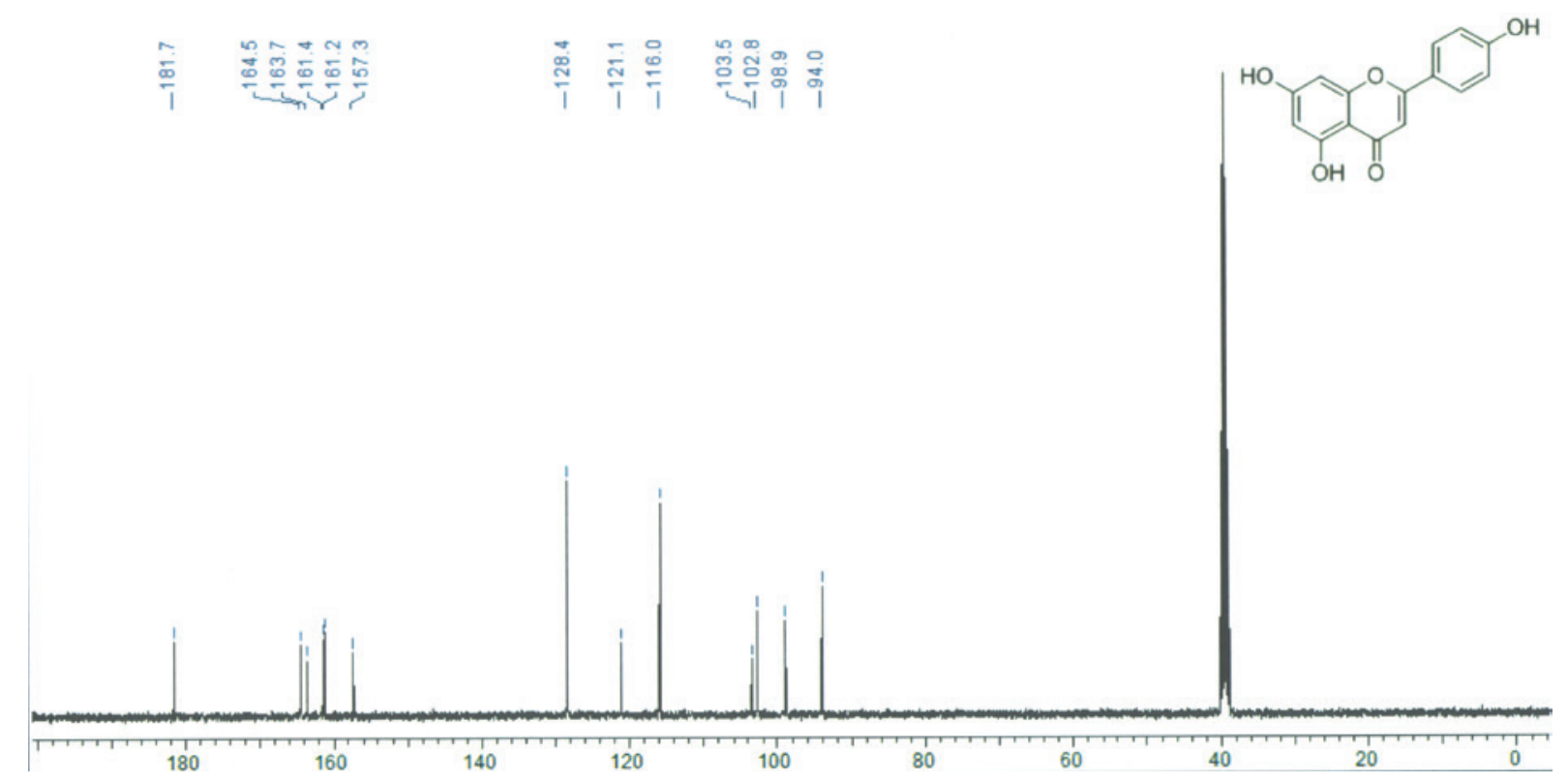

Figure 3: The ${ }^{13} \mathrm{C}$ NMR (DMSO- $\mathrm{d}_{6}, 100 \mathrm{MHz}$ ) of synthesized apigenin.

considered as a single fruit from the commercial point of view, from the botanical point of view they are different. In these experiments, fresh albedos were separated and this helped determine the percentage of each constituent in the fruit (Table 1). We also determined the weight loss in albedo drying, which is $24.4 \%$ after 2 days of air-drying followed by oven drying overnight.

Our preliminary experiments were performed with the room temperature extraction of dry albedo with methanol for 3 days, followed by the filtration of the methanolic extract, and the evaporation of the solvent under reduced pressure at $45^{\circ} \mathrm{C}$ in a rotary evaporator. Water was added to the dry extract, and the obtained mixture was heated at $70^{\circ} \mathrm{C}$ for $30 \mathrm{~min}$ before being transferred into an Erlenmeyer flask. The dichloromethane was added and the mixture was swirled and set aside until total crystallization was observed (2-4 days). The organic layer was pulled off and crystals were collected by filtration and dried in a desiccator on vacuum.

The experiments showed that the replacement of dry albedo from pomelo for grapefruit resulted in similar yields of naringin extraction with those mentioned in the literature $(2.4 \%-2.2 \% \mathrm{w} / \mathrm{w}$, respectively) (Method A, Table 2). In order to improve the yields and time required, we proposed a more efficient methanol extraction at $55^{\circ} \mathrm{C}$ for $1 \mathrm{~h}$, followed by the solvent separation, and new extraction. Both the solvent evaporation of combined organic phases and crystallization were performed in a similar procedure. In addition to the decrease in extraction time, which resulted in a 3-day shorter process, an improvement of $18 \%$ in the overall yield was observed, which reached 2.6\% w/w (Method B, Table 2). Encouraged

Table 1: Grapefruit composition data and albedo weight loss upon drying.

\begin{tabular}{|c|c|c|c|c|c|c|c|c|c|}
\hline \multirow[t]{2}{*}{ Entry } & \multirow[t]{2}{*}{ Fruit weight (g) } & \multicolumn{2}{|c|}{ Fresh albedo } & \multicolumn{2}{|c|}{ Dry albedo } & \multicolumn{2}{|c|}{ Flavedo } & \multicolumn{2}{|r|}{ Pulp } \\
\hline & & Weight (g) & $(\%)^{\mathrm{a}}$ & Weight (g) & $(\%)^{b}$ & Weight (g) & $(\%)^{\mathrm{a}}$ & Weight (g) & $(\%)^{a}$ \\
\hline 1 & 274.67 & 28.83 & 10.5 & 8.86 & 30.7 & 48.20 & 17.5 & 197.64 & 72.0 \\
\hline 2 & 293.35 & 28.32 & 10.0 & 7.17 & 24.4 & 51.80 & 17.7 & 212.23 & 72.3 \\
\hline 3 & 301.45 & 30.89 & 10.2 & 7.97 & 25.8 & 48.44 & 16.1 & 222.12 & 73.7 \\
\hline 4 & 324.33 & 35.32 & 10.9 & 9.21 & 26.1 & 55.67 & 17.2 & 233.34 & 71.9 \\
\hline 5 & 361.01 & 56.38 & 15.6 & 11.62 & 20.6 & 57.44 & 15.9 & 247.19 & 68.5 \\
\hline 6 & 373.58 & 40.45 & 10.8 & 7.71 & 19.0 & 50.54 & 13.5 & 282.59 & 75.7 \\
\hline Average & 321.40 & 36.70 & 11.3 & 8.76 & 24.4 & 52.02 & 16.3 & 232.52 & 72.3 \\
\hline
\end{tabular}

aPercentage of total grapefruit mass.

bPercentage of dry albedo mass in relation to fresh albedo. 
Table 2: Yields (\%) of naringin extraction by different methods.

\begin{tabular}{lrrr}
\hline Entry & Method A $^{\mathrm{a}}$ & Method B $^{\mathrm{a}}$ & Method C $^{\mathrm{b}}$ \\
\hline 1 & 2.5 & 2.8 & 4.2 \\
2 & 2.1 & 2.4 & 3.9 \\
3 & 2.1 & 2.7 & 4.3 \\
Average & 2.2 & 2.6 & 4.1 \\
\hline
\end{tabular}

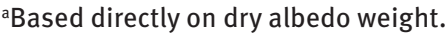

${ }^{\mathrm{b}}$ Based on dry albedo and estimated from fresh albedo weighed by $24.4 \% \mathrm{w} / \mathrm{w}$.

by the reasonable improvement in mass extraction of naringin and the shortened duration of the whole process, we decided to perform the extraction of natural product on fresh albedos recently separated from flavedos, thus avoiding the 3 day-drying step. After $2 \mathrm{~h}$ of the hot methanol extraction process, we significantly reduced the time required from 10 to 3-4 days, depending on the recrystallization time. However, the developed process brought benefits on the time and on yield of isolated product, requiring a short extraction-crystallization method C (Table 2), which could provide naringin in $4.1 \% \mathrm{w} / \mathrm{w}$ (based on $24.4 \% \mathrm{w} / \mathrm{w}$ loss of mass from fresh to dry albedo) (Table 3 ).

Table 3: Extraction data from fresh albedo in Method C.

\begin{tabular}{lrrr}
\hline $\begin{array}{l}\text { Fresh albedo } \\
\text { weight (g) }\end{array}$ & $\begin{array}{r}\text { Calculated dry albedo } \\
\text { weight }(\mathrm{g})(\mathbf{2 4 . 4 \%} \text { w/w) }\end{array}$ & $\begin{array}{r}\text { Naringin mass } \\
\text { extracted (g) }\end{array}$ & $\begin{array}{r}\text { Yield \% } \\
\text { (dry based) }\end{array}$ \\
\hline 30.01 & 7.32 & 0.3081 & 4.2 \\
71.96 & 17.56 & 0.6764 & 3.9 \\
69.84 & 17.04 & 0.7375 & 4.3 \\
\hline
\end{tabular}

This means an improvement of about $70 \%$ in relation to the method described in the literature, which is used as the basis in this work.

To evaluate the obtained naringin, we also performed its transformation into apigenin, a natural product with several biological properties and higher economic value. Transformation from one natural product to another is found in the articles of Li et al. and Oyama and Kondo, apart from some chemical synthesis described [10-14]. While Oyama and Kondo employed DDQ in refluxing 1,4-dioxane as the oxidant agent, Li et al. proposed dehydrogenation with $\mathrm{I}_{2} /$ pyridine. Of the two, the former methodology is faster and more efficient, with only the reverse hydrolysis-oxidation order. Thus, the oxidation of natural extracted naringin with iodide in pyridine at $95^{\circ} \mathrm{C}$ for $5 \mathrm{~h}$ generated rhoifolin with an $86 \%$ yield (Figure 4). A simple acid hydrolysis in hot ethanol allowed apigenin synthesis with an $86 \%$ yield ( $74 \%$ yield in two steps). This is a more efficient process than the already described oxidationhydrolysis sequence.

\section{Conclusion}

In this communication, we proposed a way by which to improve the method of isolating naringin from grapefruit peels. The modification of the known process, which involves extraction with methanol and crystallization in water with the addition of dichloromethane, allowed shorter times (reduction from 10 to 3-4 days) and higher yields (from $2.4 \%$ to $4.1 \% \mathrm{w} / \mathrm{w}$ ) through direct hot extraction with methanol using fresh albedos. To evaluate the
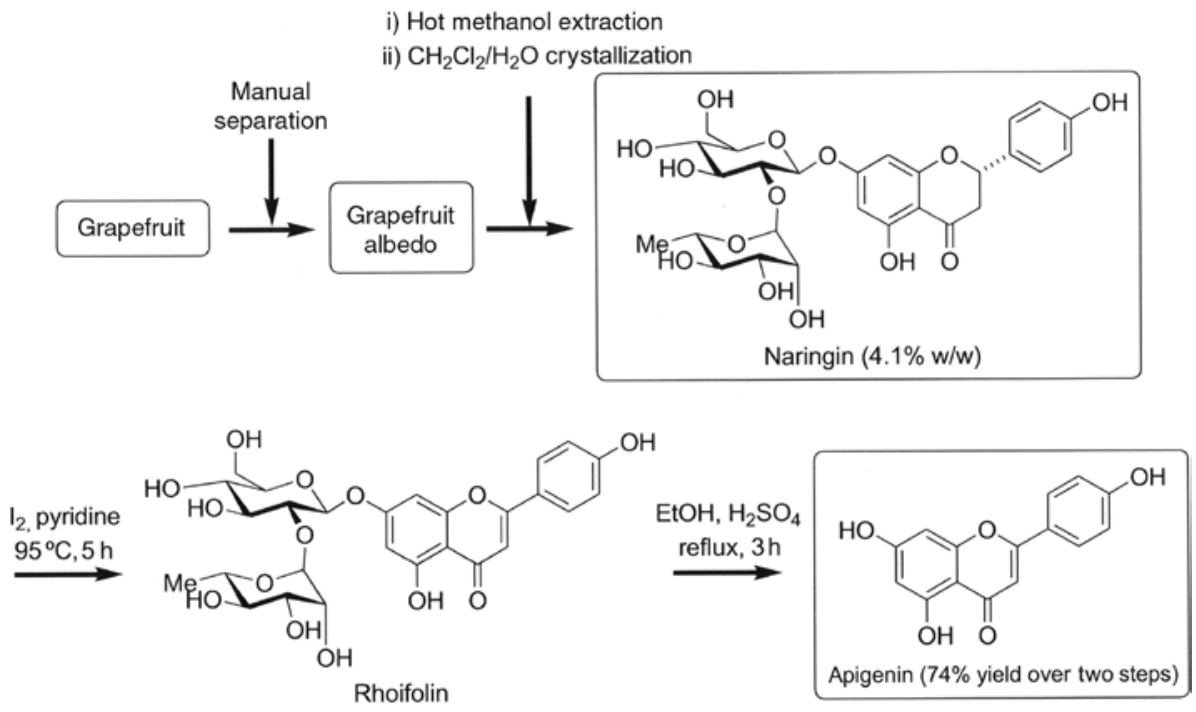

Figure 4: The extraction of naringin and its transformation into apigenin. 
product obtained, it was transformed into apigenin following a known methodology with a slight modification in the order reactional steps. The proposed transformation procedure generated higher and better yields.

Acknowledgments: The authors are grateful to the Brazilian Agencies CNPq (National Council for Scientific and Technological Development), FAPESB (Baiana Foundation for the Support of Scientific and Technological Development), and INCT E\&A (National Institute for Science and Technology for Energy and Environment) for financial support. The authors also thank Prof. Kleber Thiago de Oliveira (UFSCar) and Cláudio Viegas Jr. (UNIFAL) for conducting NMR spectra.

\section{References}

[1] Hargreaves JC, Adl ML, Warman PR. Agric. Ecosyst. Environ. 2008, 123, 1-14.
[2] Patil RH, Babu RL, Kumar MN, Kumar KMK, Hedge SM, Nagesh R, Ramesh GT, Sharma SC. Inflammation 2016, 39, 138-147.

[3] Xu M, Wang S, Song YU, Yao J, Huang K, Zhu X. Oncol. Lett. 2016, 11, 3075-3080.

[4] Shukla S, Gupta S. Pharm. Res. 2010, 27, 962-978.

[5] Magiera S, Kwietniowska E. Food Chem. 2016, 211, 227-234.

[6] Li Y, Cai S, He K, Wang Q. J. Chem. Res. 2014, 38, 287-290.

[7] Oyama KI, Kondo T. Tetrahedron 2004, 60, 2025-2034.

[8] Sudto K, Pornpakakul S, Wanichwecharungruang S. Int. J. Food Sci. Tech. 2009, 44, 1737-1742.

[9] Riaz T, Abbasi MA, Rehman AU, Shazadi T, Khan KM, Ashraf M, Ejaz SA. Asian J. Chem. 2013, 25, 1927-1933.

[10] Matsuda H, Morikawa T, Toguchida I, Yoshikawa M. Chem. Pharm. Bull. 2002, 50, 788-795.

[11] Seijas JA, Vázquez-Tato SJ, Carballido-Reboredo R. J. Org. Chem. 2005, 70, 2855-2858.

[12] Bovicelli P, D’Angelo V, Collalto D, Verzina A, D’Antona N, Lambusta D. J. Pharm. Pharmacol. 2007, 59, 1697-1701.

[13] Zhang J, Liu M, Cui W, Yang J, Liao XI. J. Chem. Res. 2013, 37, 694-696.

[14] Wang Q, Cui W, Liu M, Zhang J, Liao RQ, Liao XI, Yang J. J. Chem. Res. 2015, 39, 67-69. 